\title{
New Multiple Sclerosis Phenotypic Classification
}

\author{
Fred D. Lublin
}

Icahn School of Medicine, Corinne Goldsmith Dickinson Center for Multiple Sclerosis, Mount Sinai, New York, N.Y., USA

\section{Key Words}

Disease activity · Disease progression - Multiple sclerosis, phenotypes

\begin{abstract}
Background: In 1996, the clinical course of multiple sclerosis (MS) was characterized as relapsing-remitting, primary progressive, secondary progressive or progressive relapsing. Since then, an increased understanding of MS and its pathology prompted a re-examination of these clinical phenotypes. Main recommendations of the 2013 revisions are provided herein. Summary: Clinically isolated syndrome has been added, and progressive relapsing MS has been eliminated, from the clinical course descriptions. All forms of MS should be further subcategorized as either active or non-active. Active MS is defined as the occurrence of clinical relapse or the presence of new T2 or gadolinium-enhancing lesions over a specified period of time, preferably at least one year. An additional subcategory for patients with progressive MS differentiates between those who have shown signs of disability progression over a given time period and those who have remained stable. The term 'worsening' is recommended to describe patients whose disease is advancing for any reason, whereas 'disease progression' should be reserved for
\end{abstract}

those with progressive disease who are truly progressing (as opposed to worsening from a relapse). The term 'benign' should be used with caution as the course of MS can worsen at any time, even after many years of apparent stability. Key Messages: Newer characterizations of MS phenotypes include a consideration of disease activity (based on the clinical relapse rate and imaging findings) and disease progression. Accurate clinical course descriptions are useful for communication, prognostication, clinical trial design and to guide everyday clinical decision-making.

(c) 2014 S. Karger AG, Basel

\section{Introduction}

In 1996, a consensus paper was published in which four clinical courses of multiple sclerosis (MS) were defined: relapsing-remitting MS, primary progressive MS, secondary progressive MS and progressive-relapsing MS [1]. These distinctions were determined by polling members of the international MS clinical community. The characterizations were rapidly adopted into practice and served to better communicate a patient's clinical course and to define clinical trial populations. At the time, no biomarkers or magnetic resonance imaging (MRI) sig-

\section{KARGER}

E-Mail karger@karger.com

www.karger.com/ene (c) 2014 S. Karger AG, Basel

0014-3022/14/0721-0001\$39.50/0
Dr. F.D. Lublin

Corinne Goldsmith Dickinson Center for Multiple Sclerosis Icahn School of Medicine at Mount Sinai

5 East 98th Street, Box 1138, New York, NY 10029-6574 (USA)

E-Mail fred.lublin@mssm.edu 
nals were available to distinguish between the different MS clinical courses.

Since then, an increased understanding of MS and its pathology, together with general concern that these descriptors may no longer adequately reflect recently identified clinical aspects of the disease, prompted a re-examination of MS disease phenotypes. The project was carried out under the auspices of the Clinical Trials Committee, and was jointly sponsored by the International Advisory Committee on Clinical Trials in Multiple Sclerosis of the European Committee for Treatment and Research in MS (ECTRIMS) and the US National Multiple Sclerosis Society (NMSS). Additional support was provided by the Americas Committee for Treatment and Research in MS (ACTRIMS), the Multiple Sclerosis International Federation (MSIF) and the Multiple Sclerosis Society of Canada (MSSC).

Some specific tasks of the undertaking were to:

- Improve clinical descriptive terminology

- Assess the value of MRI and other imaging techniques in discriminating between clinical courses

- Assess the feasibility/value of fluid biomarkers in discriminating between clinical courses

- Evaluate other assays, including electrophysiology.

Another major goal was to identify and recommend research strategies to move the evaluation of MS phenotypes forward in areas where data and/or consensus are lacking.

The 2013 revisions were published online in May 2014 [2]. A brief summary of the main conclusions is provided herein.

\section{Defining the Clinical Course of Multiple Sclerosis: The 2013 Revisions}

\section{Core Phenotypes and Modifiers}

The core MS phenotypes (relapsing-remitting and progressive disease) described in 1996 have been retained, although with some modifications.

Assessment of disease activity as measured by clinical relapses or central nervous system (CNS) lesion activity, and assessment of ongoing progression of disability, are regarded as important modifiers of the core phenotypes.

Primary progressive MS is not considered as a separate entity but part of the spectrum of progressive disease. Pathologically, inflammatory changes are observed throughout the CNS in all forms of MS with differences between phenotypes being quantitative rather than qualitative.
The progressive-relapsing MS phenotype has been eliminated and is now categorized as 'primary progressive MS with activity'.

Clinically isolated syndrome (CIS) is considered to be part of the spectrum of MS phenotypes and should be followed to determine subsequent disease course. The definition of CIS as derived from other sources has been used without modification.

Radiologically isolated syndrome (RIS) is not considered to be part of the spectrum of MS phenotypes as patients lack clinical signs and symptoms and MRI findings alone are insufficient to establish a diagnosis of MS.

\section{Terminology}

To enhance clarity, it is recommended that the term 'worsening' be used in preference to 'progressing' to describe individuals whose disease is advancing due to frequent relapses or incomplete relapse recovery (as measured on any clinical scale such as the Expanded Disability Status Scale [EDSS], walking time etc.). The term 'progression' is to be reserved for individuals in a progressive phase of MS with evidence of gradual worsening over time. To illustrate the difference, a patient with stepwise accumulation of deficit due to acute attacks is worsening, whereas a patient who has entered the secondary progressive phase of MS (e.g. graduated from a cane to a walker) is progressing (and also worsening).

It is recommended that the term 'confirmed worsening' over a defined period of time be used in preference to 'sustained worsening' as the latter term infers a state of permanence which is often not the case; this recommendation is particularly relevant in the clinical trials setting.

It is recommended that the terms 'benign' and 'malignant' to describe disease course be used with caution. In particular, benign is not a definitive term; it is a retrospective diagnosis and may be deceiving in some cases as MS can worsen at any stage, even after long periods (e.g. 10 or 20 years) of apparent stability.

\section{Imaging and Biological Markers}

As in 1996, there are no advanced MRI metrics or fluid biomarkers that can reliably predict the MS disease course.

\section{MS Phenotype Descriptions}

Despite the lack of MRI metrics to distinguish between clinical phenotypes, refined descriptors are proposed that include consideration of disease activity, 
based on clinical relapse rate and imaging findings, and disease progression. Among the perceived values of the newer characterizations is to guide inclusion criteria for clinical trials. A successful study outcome in a homogeneous group of patients can increase confidence about a specific patient type most likely to respond to a particular intervention.

\section{Relapsing-Remitting Disease}

CIS is a clear-cut syndrome such as optic neuritis, brain stem/cerebellar dysfunction or partial myelitis and is now considered to be part of the relapsing-remitting MS disease spectrum (fig. 1). CIS may be active or not active. To be classified as active, a clinical or radiological event (gadolinium-enhancing $(\mathrm{Gd}+)$ or new/enlarging T2 lesions) must follow the CIS. For example, an episode of partial myelitis in the absence of MRI activity is considered to be 'CIS not active' and would maintain that designation until development of the next episode or MRI change. In turn, 'CIS active' may or may not fulfill criteria (e.g. McDonald 2010 [3]) for relapsing-remitting MS. Classification as relapsing-remitting MS would require MRI evidence of dissemination in space as well as $\mathrm{Gd}+$ and non-enhancing T2 lesions on a single MRI scan and/ or a subsequent event.

Relapsing-remitting MS is also characterized as active or not active within a specified time frame (e.g. 6 months, 1 year) (fig. 1). The actual time frame can be an individual decision based on usual clinical practice but must be specified. As a guide, assessments for disease activity should be conducted at least annually.

\section{Progressive Disease}

Progressive disease, whether primary progressive or secondary progressive, has four possible sub-classifications taking into account the level of disability (fig. 2):

- Active and with progression (individual has had an attack and is also gradually worsening)

- Active but without progression (e.g. individual has had an attack within a previous specified timeframe, i.e. 1 year, 2 years)

- Not active but with progression (e.g. walking speed has decreased)

- Not active and without progression (stable disease).

An impetus for developing these characterizations in progressive disease was clinical trial evidence suggesting that a proportion of individuals with primary progressive MS have relapses and/or Gd+ lesions [4, 5]. Identification of this group was considered important because, theo-

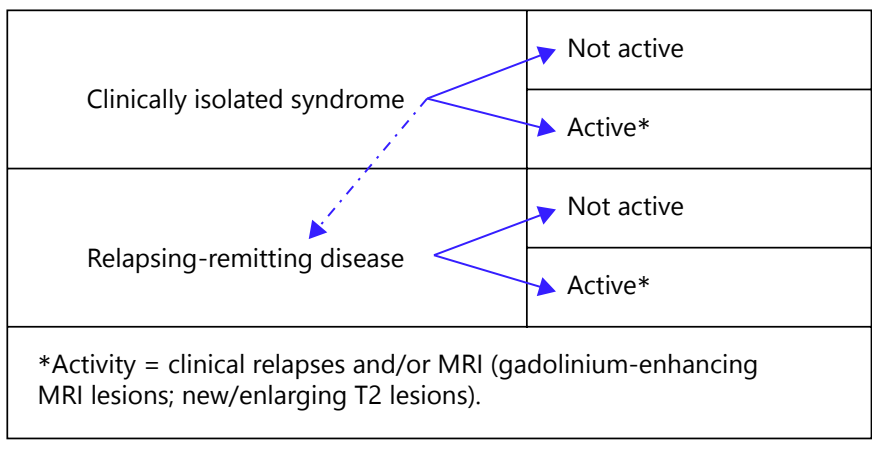

Fig. 1. 2013 multiple sclerosis phenotype descriptions: relapsingremitting disease.

\begin{tabular}{|c|c|}
\hline $\begin{array}{l}\text { Primary progressive } \\
\text { (progressive accumulation } \\
\text { of disability from onset) }\end{array}$ & Active* and with progression ${ }^{\#}$ \\
\hline \multirow[b]{2}{*}{ Progressive disease } & Active* but without progression \\
\hline & Not active but with progression $\#$ \\
\hline $\begin{array}{c}\checkmark \\
\text { Secondary progressive } \\
\text { (progressive accumulation } \\
\text { of disability after } \\
\text { an initial relapsing course) }\end{array}$ & $\begin{array}{l}\text { Not active and without progression } \\
\text { (stable disease) }\end{array}$ \\
\hline \multicolumn{2}{|c|}{$\begin{array}{l}\text { *Activity = clinical relapses and/or MRI (gadolinium-enhancing } \\
\text { MRI lesions; new/enlarging T2 lesions). }\end{array}$} \\
\hline
\end{tabular}

Fig. 2. 2013 multiple sclerosis phenotype descriptions: progressive disease.

retically, a progressive patient with activity may respond differently to a disease-modifying therapy than a progressive patient without activity.

\section{Definitions}

\section{Active Disease}

Active disease can be defined individually over ' $n$ ' time; e.g. 1 year.

- Clinical: relapses, acute or sub-acute episodes of new or increasing neurological dysfunction followed by full or partial recovery, in the absence of fever or infection or evidence of a pseudo-relapse. 
- Imaging (MRI): occurrence of contrast-enhancing T1 hyperintense lesions or new or unequivocally enlarging T2 hyperintense lesions. The term 'unequivocally' has been added to highlight the importance of aligning successive scans as closely as possible when gauging enlarging lesions.

\section{Progressive Disease}

Progressive disease can be defined over ' $n$ ' time, e.g. 1 year.

- Clinical: steadily increasing objectively documented neurological dysfunction/disability without unequivocal recovery; fluctuations and phases of stability may occur.

- Imaging (MRI): no standardized imaging measures of disease progression are currently established; however, measures such as an increasing number and volume of T1 hypo-intense lesions, brain volume loss and changes in magnetization transfer imaging and diffusion tensor imaging are being explored.

\section{Worsening, Progression and Confirmed Worsening}

Worsening disease is defined as a documented increase in neurological dysfunction/disability as a result of relapses or progressive disease or both.

Disease progression is reserved solely for patients in a progressive phase of MS.

Confirmed progression or worsening describes an increase in neurological dysfunction confirmed throughout a defined time interval (e.g. 3, 6 or 12 months); this distinction is particularly relevant in the clinical trial setting. Given that neurological dysfunction may improve (especially in relapsing-remitting MS) even if initially confirmed, it is recommended that the term 'sustained' be abandoned unless disease has been measured over a long period of time.

\section{Future Research Needs}

An important aspect of the 2013 revisions to the phenotypic classification of MS was to identify future needs in MS research. Some recommended research strategies include:

- Conduct long-term longitudinal studies to standardize evaluation of individuals with MS. Specific areas for investigation include the potential for associating imaging outcomes (objective criteria) with clinical phenotypes and defining the transition between dis- ease subtypes over time. For example, at what point does secondary progressive MS occur? Is it a gradual transition from relapsing-remitting MS? Is there a durable metric that can define secondary progressive MS?

- Maintain close follow-up (clinical/imaging) of RIS to better detect subtle clinical signs/symptoms related to MS.

- Investigate a wide variety of time frames (e.g. other than annual) to assess disease activity.

- Conduct cohort studies to determine whether the severity of a clinical attack or imaging activity is a factor in mid- to long-term outcomes and to assess whether the degree of recovery from relapses has an impact on long-term outcomes and phenotype.

- Examine imaging studies of tissue damage (e.g. brain atrophy, black hole evolution, retinal nerve fibre layer thinning, other optical coherence tomography measures) and the possible relationship with clinical phenotype.

- Conduct cohort studies in large datasets to determine the potential for fluid-borne (blood, CNS) biomarkers to better define MS phenotypes.

- Investigate the contribution of standard electrophysiological assessments (evoked potentials) to clinical phenotype.

- Examine the role of patient-reported outcomes in refining clinical phenotype.

\section{Potential Uses for MS Phenotype Characterisations}

The 2013 revisions to the characterizations of MS phenotype are expected to have several important applications. These include:

- Characterization of clinical course to identify where an individual is placed within the MS disease spectrum.

- To guide study inclusion criteria to ensure better defined and more homogenous patient populations.

- As study outcome measures (e.g. time to activity; time to progression).

- To evaluate the adequacy of therapy.

- To guide the design of new studies.

- To guide the design of biomarker studies, e.g. examining the genetics of clinical course, disease severity. For example, the 200+ MS susceptibility genes range across the entire disease spectrum; however, it is possible that separate severity genes can define individuals with mild versus severe MS.
Lublin 


\section{Acknowledgments}

The MS Phenotype Group - 2012/2013

Fred D. Lublin, New York City, N.Y., USA; Stephen C. Reingold, Salisbury, Conn., USA; Jeffrey A. Cohen, Cleveland, Ohio, USA; Gary R. Cutter, Birmingham, Ala., USA; Per Soelberg Sørensen, Copenhagen, Denmark; Alan J. Thompson, London, UK; Jerry S. Wolinsky, Houston, Tex., USA; Laura J. Balcer, New York City, N.Y., USA; Brenda Banwell, Philadelphia, Pa., USA; Frederik Barkhof, Amsterdam, The Netherlands; Bruce Bebo Jr, Portland, Oreg., USA; Peter A. Calabresi, Baltimore, Md., USA; Michel Clanet, Toulouse, France; Giancarlo Comi, Milan, Italy; Robert J. Fox, Cleveland, Ohio, USA; Mark S. Freedman, Ottawa, Ont., Canada; Andrew D. Goodman, Rochester, N.Y., USA; Matilde Inglese, New York City, N.Y., USA; Ludwig Kappos, Basel, Switzerland; Bernd C. Kieseier, Düsseldorf, Germany; John A. Lincoln, Houston, Tex., USA; Catherine Lubetzki, Paris, France; Aaron E. Miller, New York City, N.Y., USA; Xavier Montalban, Barcelona, Spain; Paul W. O'Connor, Toronto, Ont., Canada; John Petkau, Vancouver, B.C., Canada; Carlo Pozzilli, Rome, Italy; Richard A Rudick, Cleveland, Ohio, USA; Maria Pia Sormani, Genoa, Italy; Olaf Stüve, Dallas, Tex., USA; Emmanuelle Waubant, San Francisco, Calif., USA; Chris H. Polman, Amsterdam, The Netherlands.

\section{Disclosures/Conflict of Interest}

FDL has received research funding from Acorda Therapeutics Inc; Biogen Idec; Celgene; Genzyme; National Institute of Health (NIH); National Multiple Sclerosis Society (NMSS), Novartis Pharmaceuticals Corp; Sanofi; Teva Neuroscience Inc. He has served as an advisor or consultant for: Acorda Therapeutics; Actelion Pharmaceuticals Ltd; Bayer Healthcare Pharmaceuticals; Biogen Idec Inc; Bristol-Myers Squibb Company; Celgene Corporation; Coronado Biosciences; EMD Serono Inc; Forward Pharma A/S; Genentech Inc; Genzyme Corporation; Johnson \& Johnson Pharmaceutical Research \& Development L.L.C.; Medimmune Inc; Novartis Pharmaceuticals Corporation; Receptos Inc; Questcor Pharmaceuticals Inc; Revalesio Corporation; Roche; Sanofi; Teva Neuroscience Inc; XenoPort Inc; FDL is cochief editor of Multiple Sclerosis and Related Diseases and owns stock, stock options, or bonds from Cognition Pharmaceuticals, Inc.

Writing assistance was provided by Content Ed Net (Madrid, Spain), with funding from Laboratorios Almirall SA (Barcelona, Spain).

\section{References}

1 Lublin FD, Reingold SC: Defining the clinical course of multiple sclerosis: results of an international survey. National Multiple Sclerosis Society (USA) Advisory Committee on Clinical Trials of New Agents in Multiple Sclerosis. Neurology 1996;46:907-911.

$>2$ Lublin FD, Reingold SC, Cohen JA, Cutter GR, Sørensen PS, Thompson AJ, Wolinsky JS, Balcer LJ, Banwell B, Barkhof F, Bebo B Jr, Calabresi PA, Clanet M, Comi G, Fox RJ, Freedman MS, Goodman AD, Inglese $M$, Kappos L, Kieseier BC, Lincoln JA, Lubetzki C, Miller AE, Montalban X, O'Connor PW, Petkau J, Pozzilli C, Rudick RA, Sormani MP, Stüve O, Waubant E, Polman CH: Defining the clinical course of multiple sclerosis: the 2013 revisions. Neurology 2014;83:278-286.
Polman CH, Reingold SC, Banwell B, Clanet M, Cohen JA, Filippi M, Fujihara K, Havrdova E, Hutchinson M, Kappos L, Lublin FD, Montalban X, O'Connor P, Sandberg-Wollheim M, Thompson AJ, Waubant E, Weinshenker B, Wolinsky JS: Diagnostic criteria for multiple sclerosis: 2010 revisions to the McDonald criteria. Ann Neurol 2011;69:292302.
4 Wolinsky JS, Narayana PA, O'Connor P, Coyle PK, Ford C, Johnson K, Miller A, Pardo L, Kadosh S, Ladkani D; PROMiSe Trial Study Group: Glatiramer acetate in primary progressive multiple sclerosis: results of a multinational, multicenter, double-blind, placebocontrolled trial. Ann Neurol 2007;61:14-24.

5 Hawker K, O'Connor P, Freedman MS, Calabresi PA, Antel J, Simon J, Hauser S, Waubant E, Vollmer T, Panitch H, Zhang J, Chin $\mathrm{P}$, Smith CH; OLYMPUS trial group: Rituximab in patients with primary progressive multiple sclerosis: results of a randomized double-blind placebo-controlled multicenter trial. Ann Neurol 2009;66:460-471. 